\title{
On Some Embedment of Groups into Wreath Products
}

\author{
Enoch Suleiman (1), Muhammed Salihu Audu \\ ${ }^{1}$ Department of Mathematics, Federal University Gashua, Yobe State, Nigeria \\ ${ }^{2}$ Department of Mathematics, University of Jos, Jos, Nigeria \\ Email: enochsuleiman@gmail.com, audumso2@gmail.com
}

How to cite this paper: Suleiman, E. and Audu, M.S. (2021) On Some Embedment of Groups into Wreath Products. Advances in Pure Mathematics, 11, 109-120. https://doi.org/10.4236/apm.2021.112007

Received: December 22, 2020

Accepted: February 2, 2021

Published: February 5, 2021

Copyright $\odot 2021$ by author(s) and Scientific Research Publishing Inc. This work is licensed under the Creative Commons Attribution International License (CC BY 4.0).

http://creativecommons.org/licenses/by/4.0/

Open Access

\begin{abstract}
In this paper, we showed how groups are embedded into wreath products, we gave a simpler proof of the theorem by Audu (1991) (see [1]), also proved that a group can be embedded into the wreath product of a factor group by a normal subgroup and also proved that a factor group can be embedded inside a wreath product and the wreath product of a factor group by a factor group can be embedded into a group. We further showed that when the abstract group in the Universal Embedding Theorem is a p-group, cyclic and simple, the embedding becomes an isomorphism. Examples were given to justify the results.
\end{abstract}

\section{Keywords}

Wreath Product, Direct Product, Homomorphism, Embedding, p-Group, Cyclic, Simple

\section{Introduction}

Embedding is one of the most important properties of wreath product; this property was further investigated with regards to imprimitivity of groups, normal subgroups and Quotient Group. Many people have worked on wreath products over the years and their work is as shown below:

Suzuki [2] in 1982 proved the Kaloujnine-Krasner Theorem that states that if $F$ is a group extension of $N$ by $G$, then $F$ can be embedded inside the standard wreath product $N \mathrm{Wr} G$. Audu [1] in 1991 proved that a permutation group that is transitive and imprimitive that is acting on a finite set can be embedded inside the wreath product $X^{\Delta} w r G^{\Delta}$, where $X=G_{\{\Delta\}}$ is the setwise stabilizer of $G$ and $X^{\Delta}, G^{\Delta}$ are the constituents of $X$ and $G$ respectively while $\Delta$ is an element in the set of imprimitivity. Dixon \& Mortimer [3] in 1996 expounded 
that any transitive and imprimitive group $G$ can be embedded inside a wreath product in such a way that the kernel consists of the set of elements of the group which are mapped into the base group. Conway et al. [4] in 1998 gave an example of a group of degree 8 that is generated by $x=(15)(37), y=(1458)(23)(67)$ and $z=(02)(13)(46)(57)$ with four block system namely:

$\Delta_{1}=\{\{0,4\},\{1,5\},\{2,6\},\{3,7\}\}, \Delta_{2}=\{\{0,3,4,7\},\{1,2,5,6\}\}$, $\Delta_{3}=\{\{0,2,4,6\},\{1,3,5,7\}\}, \Delta_{4}=\{\{0,1,4,5\},\{2,3,6,7\}\}$. They showed that the group which is imprimitive can be embedded inside the wreath product $\Delta_{1}$ wr $E(4)$.

Bamberg [5] in 2005 states that if $G$ is any transitive imprimitive permutation group on a set $\Omega$ and $\Lambda$ a $G$-invariant partition of $\Omega$, if also $\Delta$ is an element of $\Lambda$ and $C$ the permutation group induced by the action of $G_{\{\Delta\}}$ on $\Delta$. If $D$ is the group of permutations induced by $G$ on $\Lambda$, then $\Omega$ may be identified with $\Delta \times \Lambda$ in such a way that $G$ can be embedded into the wreath product $C$ wr $D$ in imprimitive action. Bamberg further states that if $G$ is transitive but imprimitive group on a finite set $\Omega$, then $G$ can be embedded into the wreath product $G_{\{\Delta\}}^{\Delta} w r S_{n}$ acting in imprimitive action, where $\Delta$ is a block for $G, G_{\{\Delta\}}^{\Delta}$ is the group induced by the action of setwise stabilizer $G_{\{\Delta\}}$ on $\Delta$, and $n$ is the size of the orbit of $\Delta$ under $G$. If $G_{\{\Delta\}}^{\Delta}$ is also imprimitive, then $G_{\{\Delta\}}^{\Delta}$ embeds into a wreath product. As $\Omega$ is finite, the process can continue until an embedding of $G$ into iterated wreath product of primitive groups was found. Chan [6] in 2006 proved that every faithful group action that is transitive and imprimitive is embeddable in a wreath product. Cameron [7] in 2013 showed that if $H$ is a permutation group induced on a part by its setwise stabilizer and if $K$ is the permutation group induced on the set of parts by the group $G$, then $G$ is embedded in the wreath product $H$ wr K .

Tamuli [8] in (1972) gave a new prove of the Universal Embedding Theorem and further proved that if $N$ is a subgroup of a group $H$ and all are subgroups of another group $G$, then $G$ can be embedded inside the wreath product $H w r(G / N)$. Tamuli further proved that if the subgroup $H$ has a transversal $T$ which centralizes $H$ in $G$, then the embedding $\beta: G \rightarrow H w r(G / N)$ is an extension of the diagonal embedding $\alpha_{0}: N \rightarrow N w r(G / N)$. Tamuli also proved that if $Q$ is an amalgam of two subgroups $A$ and $B$ in which their intersection $N$ is a normal subgroup of $B$, and if $T$ is the transversal of $N$ in $B$, then the amalgam $Q$ can be embedded inside the wreath product $A w r(B / H)$. Dixon \& Mortimer [3] (1996) stated and gave a new proof of the Universal Embedding Theorem that states: If $G$ is an arbitrary group with a normal subgroup $N$, and $K:=G / N$ the factor group of $G$ by $N$, then $\phi: G \rightarrow N$ wr $K$ is an embedding such that $\phi$ maps $N$ onto $\operatorname{Im} \phi \cap B$, where $B$ is the base group of $N$ wr K . Mikaelian [9] in 2002 showed that every extension of a group $G$ where the group product is the product variety that consists of all extensions of groups, if $N$ is a normal subgroup and $H \cong G / N$, then every extension of $G$ can be isomorphically embedded into the wreath product $N \mathrm{Wr} \mathrm{H}$. Hulpke [10] in 2004 proved that a transitive group $G$ 
can be embedded inside the wreath product $N_{G}(A) w r G / M$ if $M$ is a normal subgroup of $G$ and $A$ any subgroup of $G$.

Given isomorphism between two groups, knowing how the first group is isomorphic to a subgroup of the other groups helps us to know the structures being preserved. Since a wreath product is a group with many subgroups, it is easily seen that to be isomorphic to a group.

In this paper, we were able to give new proof of the theorem by Audu (1991) (see [1]); we obtained the proof of the following: a group can be embedded into the wreath product of a factor group by a normal subgroup; the wreath product of two factor groups can be embedded into a group; when the abstract group in the Universal Embedding Theorem is a p-group, cyclic and simple, the embedding is an isomorphism.

\section{Basic Definitions}

An action of a group $G$ on a non-empty set $\Omega$ is a map $\mu: G \times \Omega \rightarrow \Omega$ denoted by $\mu(g, \alpha)=\alpha^{g}$ for all $g \in G, \alpha \in \Omega$ such that

$$
\begin{gathered}
\text { 1) }\left(\alpha^{g}\right)^{h}=\alpha^{g h} \text { for all } \alpha \in \Omega \text { and all } g, h \in G \\
\text { 2) } \alpha^{1}=\alpha \text { for all } \alpha \in \Omega
\end{gathered}
$$

We then say that $G$ acts on $\Omega$.

If $G$ and $H$ are groups, then $G \times H$ is a group called the Direct Product of $G$ and $H$ where $G \times H=\{(g, h) \mid g \in G, h \in H\}$ and multiplication is defined by

$$
\left(g_{1}, h_{1}\right)\left(g_{2}, h_{2}\right)=\left(g_{1} g_{2}, h_{1} h_{2}\right)
$$

If $1_{G}$ is the identity for $G$, and $1_{H}$ is the identity for $H$, then $\left(1_{G}, 1_{H}\right)$ is the identity for

$$
G \times H \text { and }(g, h)^{-1}=\left(g^{-1}, h^{-1}\right)
$$

(see details in [11])

If $\Gamma$ and $\Delta$ are nonempty sets, then we call $\Gamma^{\Delta}$ to denote the set of all functions from $\Delta$ to $\Gamma$. In the case that $C$ is a group, we turn $C^{\Delta}$ into a group by defining product "pointwise"

$$
f g(\gamma):=f(\gamma) g(\gamma)
$$

for all $f, g \in C^{\Delta}$ and $\gamma \in \Delta$ where the product in the right is in $C$.

Let $C$ and $D$ be groups and suppose $D$ acts on the nonempty set $\Delta$. Then the wreath product of $C$ by $D$ is defined with respect to this action is defined to be the semidirect product $C^{\Delta} \rtimes D=C w r D$ where $D$ acts on the group $C^{\Delta}$ via

$$
f^{d}(\gamma):=f\left(\gamma^{d^{-1}}\right)
$$

for all $f \in C^{\Delta}, \gamma \in \Delta$ and $d \in D$ and multiplication for all $\left(f_{1}, d_{1}\right),\left(f_{2}, d_{2}\right) \in C$ wr $D$ is given by

$$
\left(f_{1}, d_{1}\right)\left(f_{2}, d_{2}\right)=\left(f_{1} f_{2}^{d_{1}^{-1}}, d_{1} d_{2}\right)
$$




$$
\text { Clearly, }|C w r D|=|C|^{|\Delta|}|D|
$$

(see details in [12])

A homomorphism $\phi: G \rightarrow H$ that is one-to-one (injective) is called an embedding: the group $G$ "embeds" into $H$ as a subgroup. If $\phi$ is not one-to-one, then it is a quotient. Note that if $\phi: G \rightarrow H$ is an embedding, then $\operatorname{ker}(\phi)=\left\{e_{G}\right\}$ and from the First Isomorphism Theorem, $\operatorname{Im}(\phi) \cong G /\left\{e_{G}\right\} \cong G$. Now $\operatorname{Im}(\phi) \leq H$ as $\phi: G \rightarrow H$ is a homomorphism, and so we conclude that in an embedding, $G$ is isomorphic to a subgroup of $H$. In symbol $G \lesssim H$.

\section{Results}

We now give an alternative proof to a theorem of Audu (1991) and also outline some propositions with their proofs. We proved embedding by showing that they are homomorphic and injective. We gave three conditions on the Universal Embedding Theorem (Dixon \& Mortimer, 1996) when the group is a p-group and when the group is simple.

Theorem 1 (see [1]): Let $G$ be any transitive and imprimitivity group acting on a set $\Omega$; let $\Lambda:=\left\{\Delta_{i}: 1 \leq i \leq s\right\}$ be a system of imprimitivity of $G$ and $\Delta$ be an element of $\Lambda$. If $X:=G_{\{\Delta\}}$ then $G$ can be embedded inside the wreath product $X^{\Delta} w r G^{\Delta}$.

Proof: Let $\psi: G \rightarrow G^{\Lambda}$ be a homomorphism of $G$ onto $G^{\Lambda}$ with kernel $K:=G_{(\Lambda)}$. Let $\psi$ be defined by

$$
\psi\left(t_{u}\right)=u
$$

for each $u \in G^{\Lambda}$. If $x \in G$, then

$$
\begin{aligned}
\psi\left(t_{u} x\right) & =\psi\left(t_{u}\right) \psi(x) \\
& =u \psi(x) \quad(\text { by }(9)) \\
& =\psi\left(t_{u \psi(x)}\right) \quad(\text { by }(9))
\end{aligned}
$$

Therefore, since $\psi\left(t_{u} x\right)=\psi\left(t_{u \psi(x)}\right)$ it implies that $\psi\left(t_{u} x\right) \psi^{-1}\left(t_{u \psi(x)}\right)=1$. Therefore, $\psi\left(t_{u} x t_{u \psi(x)}^{-1}\right)=1$. Thus $t_{u} x t_{u \psi(x)}^{-1}$ lies in the kernel $K$. That is, $t_{u} x t_{u \psi(x)}^{-1} \in G_{(\Lambda)}$. By the definition of wreath product, we can define a function for each $x \in G$, such that $f_{x}: G^{\Lambda} \rightarrow X^{\Delta}$ by

$$
f_{x}(u):=t_{u} x t_{u \psi(x)}^{-1}
$$

for all $u \in G^{\Lambda}$.

We claim that $\phi(x):=\left(f_{x}, \psi(x)\right) \in X^{\Delta} w r G^{\Delta}$ defines an embedding $\phi$ of $G$ into $X^{\Delta} w r G^{\Delta}$ with the function (10).

We seek to show that $\phi$ is a homomorphism and is injective, hence an embedding.

Take $x, y \in G$, then

$$
\begin{aligned}
\phi(x) \phi(x) & =\left(f_{x}, \psi(x)\right)\left(f_{y}, \psi(y)\right) \\
& =\left(f_{x} f_{y}^{\psi(x)^{-1}}, \psi(x) \psi(y)\right) \quad(\text { by }(7))
\end{aligned}
$$


Now since $\psi$ is a homomorphism,

$$
\psi(x) \psi(y)=\psi(x y)
$$

By (10), $f_{x} f_{y}^{\psi(x)^{-1}}$ can be expressed for all $u \in G^{\Lambda}$ as follows:

$$
\begin{aligned}
f_{x}(u) f_{y}^{\psi(x)^{-1}}(u) t_{u \psi(x y)} & =f_{x}(u) f_{y}(u \psi(x)) t_{u \psi(x) \psi(y)} \quad \text { (by (6) and (11)) } \\
& =f_{x}(u) t_{u \psi(x)} t_{u \psi(x) \psi(y)}^{-1} t_{u \psi(x) \psi(y)} y \quad(\text { by }(10)) \\
& =f_{x}(u) t_{u \psi(x)} y \\
& =t_{u} x t_{u \psi(x)}^{-1} t_{u \psi(x)} y \quad(\text { by }(10)) \\
& =t_{u} x y \quad(\text { by }(10)) \\
& =f_{x y}(u) t_{u \psi(x y)}
\end{aligned}
$$

Therefore, we have that

$$
f_{x} f_{y}^{\psi(x)^{-1}}=f_{x y}
$$

Hence $\phi(x) \phi(x)=\left(f_{x y}, \psi(x y)\right)=\phi(x y)$ (by (11) and (12)). Hence $\phi$ is a homomorphism.

Next, we show that $\phi$ is injective. Now $\operatorname{ker} \phi=1$ since $\phi(x)=1$ implies $f_{x}=1$ and $\psi(x)=1$, and so $x=t_{1}^{-1} f_{x}(1) t_{1 \psi(x)}=t_{1}^{-1} t_{1}=1$. Thus $G \lesssim X^{\Delta} w r G^{\Delta}$.

\section{Example 1:}

$$
\begin{aligned}
G= & \langle(12345678)\rangle \\
\text { Let }= & \{(1),(12345678),(1357)(2468),(14725836), \quad \text { is a } \\
& (15)(26)(37)(48),(16385274),(1753)(2864),(18765432)\}
\end{aligned}
$$

transitive group. Then $\Delta=\{1,3,5,7\}$ is block of $G$.

$$
\begin{aligned}
& X=G_{\Delta}=\{(1),(1357)(2468),(15)(26)(37)(48),(1753)(2864)\} . \text { And } \\
& X^{\Delta}=(1357)=\{(1),(1357),(15)(37),(1753)\} \cong C_{4}, \\
& G^{\Delta}=(1357)=\{(1),(1357),(15)(37),(1753)\} \cong C_{4} . \text { Thus } \\
& X^{\Delta} w r G^{\Delta} \cong\langle(1234),(5678),(9101112),(13141516), \\
& (15913)(261014)(371115)(481216)\rangle
\end{aligned}
$$

order 1024.

Proposition 2: Let $G$ be an arbitrary subgroup with a normal subgroup $N$ and $\gamma: G \rightarrow G / N$ be the natural homomorphism. Suppose that $\phi: G \rightarrow G$ wr $G / N$ is a homomorphism then there is an embedding $\psi: G / N \rightarrow G w r G / N$ making the diagram

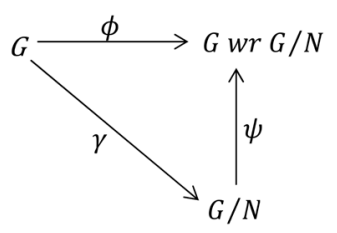

Figure 1. Commutativity diagram.

Commute. $\operatorname{ker} \psi:=\operatorname{ker} \phi / N$. 
Proof: If the diagram is to commute (see Figure 1), then we have for an arbitrary $x N \quad(x \in G)$ such that

$$
\psi(x N)=\psi \gamma(x)=\phi(x)
$$

and that is the only way it can be defined. First, we notice that (13) reposes only on the coset $x N$ and not on the representative $x$. For if, $x N=x^{\prime} N$, then $N=x^{-1} x^{\prime} N$, and $x^{-1} x^{\prime} \in N$.

$$
\text { Hence } x^{-1} x^{\prime} \in \operatorname{ker} \phi \text { and so } \begin{aligned}
& \phi\left(x^{-1} x^{\prime}\right)=1 \\
& \Rightarrow \phi\left(x^{-1}\right) \phi\left(x^{\prime}\right)=1 \\
& \Rightarrow \phi^{-1}(x) \phi\left(x^{\prime}\right)=1 \text { hence }
\end{aligned}
$$

$\phi(x)=\phi\left(x^{\prime}\right)$ and so (13) defines a map.

Next, if we have a different element of $G / N$, say $y N$, then

$$
\begin{aligned}
\psi(x N \cdot y N) & =\psi(x y N) \\
& =\phi(x y) \quad(\text { by }(13)) \\
& =\phi(x) \phi(y) \\
& =\psi(x N) \psi(y N) \quad(\text { by }(13))
\end{aligned}
$$

Thus $\psi$ is a homomorphism. From (13), $\psi(x N)=1 \Rightarrow \phi(x)=1 \Rightarrow$ $x \in \operatorname{ker} \phi$. Thus an embedding. Therefore $\operatorname{ker} \psi=\operatorname{ker} \phi / N$.

\section{Example 2:}

Let $G=S_{3}=\langle(123),(12)\rangle=\{(1),(12),(13),(23),(123),(132)\}$. $N=A_{3}=\langle(123)\rangle=\{(1),(123),(132)\}$ is a normal subgroup of $G$. The factor group $G / N=\{\{(1),(123),(132)\},\{(12),(13),(23)\}\} \cong C_{2}$. Then the wreath product

$$
\begin{array}{rl}
G & w r G / N=\langle(123),(12),(456),(45),(14)(25)(36)\rangle \\
= & (1),(56),(45),(456),(465),(46),(23),(23)(56),(23)(45),(23)(456), \\
& (23)(465),(23)(46),(12),(12)(56),(12)(45),(12)(456),(12)(465), \\
& (12)(46),(123),(123)(56),(123)(45),(123)(456),(123)(465),(123)(46), \\
& (132),(132)(56),(132)(45),(132)(456),(132)(465),(132)(46),(13), \\
& (13)(56),(13)(45),(13)(456),(13)(465),(13)(46),(14)(25)(36), \\
& (14)(2536),(1425)(36),(142536),(143625),(1436)(25),(14)(2635), \\
& (14)(26)(35),(142635),(1426)(35),(1435)(26),(143526),(1524)(36), \\
& (153624),(15)(24)(36),(1536)(24),(15)(2436),(152436),(152634), \\
& (1534)(26),(15)(2634),(153426),(15)(26)(34),(1526)(34),(163524), \\
& (1624)(35),(1635)(24),(16)(24)(35),(162435),(16)(2435),(1634)(25), \\
& (162534),(163425),(16)(2534),(1625)(34),(16)(25)(34)\}
\end{array}
$$

which is a group of order 72 .

Proposition 3: Let $G$ be an arbitrary group with a normal subgroup $N$, and put $K:=G / N$. Then there is an embedding $\phi: G \rightarrow K w r N$ such that $\phi$ maps $K$ onto $\operatorname{Im} \phi \bigcap B$ where $B$ is the base group of $K w r N$. (Thus $K w r N$ 
contains an isomorphic copy of every extension $G$ of $K$ by $N$.)

Proof: Let $\psi: G \rightarrow N$ be the natural homomorphism of $G$ onto $K$. Let $T:=\left\{t_{u} \mid u \in K\right\}$ be a set of right coset representatives of $N$ in $G$ such that

$$
\psi\left(t_{u}\right)=u
$$

for each $u \in K$. If $x \in G$,

$$
\begin{aligned}
\psi\left(t_{u} x\right) & =\psi\left(t_{u}\right) \psi(x) \\
& =u \psi(x) \quad(\text { by }(14)) \\
& =\psi\left(t_{u \psi(x)}\right) \quad(\text { by (14)) }
\end{aligned}
$$

Therefore, since $\psi\left(t_{u} x\right)=\psi\left(t_{u \psi(x)}\right)$ it implies that $\psi\left(t_{u} x\right) \psi^{-1}\left(t_{u \psi(x)}\right)=1$. Therefore, $\psi\left(t_{u} x t_{u \psi(x)}^{-1}\right)=1$. Thus $t_{u} x t_{u \psi(x)}^{-1}$ lies in $\operatorname{ker}(\psi)$. That is, $t_{u} x t_{u \psi(x)}^{-1} \in \operatorname{ker}(\psi)$. By the definition of the wreath product, we can define a function $f_{x}$ for each $x \in G$, such that $f_{x}: N \rightarrow K$ by

$$
f_{x}(u):=t_{u} x t_{u \psi(x)}^{-1}
$$

for all $u \in N$.

We claim that $\phi(x):=\left(f_{x}, \psi(x)\right) \in K w r N$ defines an embedding $\phi$ of $G$ into $K w r N$ with the function (15).

We seek to show that $\phi$ is an embedding.

Take $x, y \in G$, then

$$
\begin{aligned}
\phi(x) \phi(x) & =\left(f_{x}, \psi(x)\right)\left(f_{y}, \psi(y)\right) \\
& =\left(f_{x} f_{y}^{\psi(x)^{-1}}, \psi(x) \psi(y)\right) \quad(\text { by }(7))
\end{aligned}
$$

Now since $\psi$ is a homomorphism,

$$
\psi(x) \psi(y)=\psi(x y)
$$

By (15), $f_{x} f_{y}^{\psi(x)^{-1}}$ can be expressed for all $u \in N$ as follows:

$$
\begin{aligned}
f_{x}(u) f_{y}^{\psi(x)^{-1}}(u) t_{u \psi(x y)} & =f_{x}(u) f_{y}(u \psi(x)) t_{u \psi(x) \psi(y)} \quad \text { (by (15) and (16)) } \\
& =f_{x}(u) t_{u \psi(x)} t_{u \psi(x) \psi(y)}^{-1} t_{u \psi(x) \psi(y)} y \quad \text { (by (15)) } \\
& =f_{x}(u) t_{u \psi(x)} y \\
& =t_{u} x t_{u \psi(x)}^{-1} t_{u \psi(x)} y \quad(\text { by (15)) } \\
& =t_{u} x y \\
& =f_{x y}(u) t_{u \psi(x y)} \quad(\text { by (15)) }
\end{aligned}
$$

Therefore, we have that

$$
f_{x} f_{y}^{\psi(x)^{-1}}=f_{x y}
$$

Hence $\phi(x) \phi(x)=\left(f_{x y}, \psi(x y)\right)=\phi(x y)$ (by (16) and (17)). Hence $\phi$ is a homomorphism.

Next, we show that $\phi$ is injective. Now $\operatorname{ker} \phi=1$ since $\phi(x)=1$ implies $f_{x}=1$ and $\psi(x)=1$, and so $x=t_{1}^{-1} f_{x}(1) t_{1 \psi(x)}=t_{1}^{-1} t_{1}=1$.

Finally, $\phi(x)$ lies in $B$ when $\psi(x)=1$, and this happens exactly when 
$x \in N$. Thus $G \lesssim K w r N$.

\section{Example 3:}

Let $G=\langle(123456)\rangle$

Let $=\{(1),(123456),(135)(246),(14)(25)(36),(153)(264),(165432)\}$ and

$N=\langle(135)(246)\rangle=\{(1),(135)(246),(153)(264)\} \cong C_{3}$ is a normal subgroup of

$G$. Then

$$
K=G / N=\{\{(1),(135)(246),(153)(264)\},
$$

$$
\{(123456),(14)(25)(36),(165432)\}\} \cong C_{2} \text {. Then the wreath }
$$

product

$$
\begin{aligned}
K w r N \cong & \{(1),(56),(34),(34)(56),(12),(12)(56),(12)(34),(12)(34)(56), \\
& (135)(246),(135246),(136245),(136)(245),(146235),(146)(235), \\
& (145)(236),(145236),(153)(264),(154263),(153264),(154)(263), \\
& (164253),(163)(254),(164)(253),(163254)\}
\end{aligned}
$$

which is a group of order 24.

Proposition 4: Let $G$ be any arbitrary group with a normal subgroup $N$ and put $K:=G / N$. Then there is an embedding $\phi: K$ wr $K \rightarrow G$ such that $\phi$ maps $\operatorname{Im} \phi \cap B$ onto $K$, where $B$ is the base group of $K w r K$ and $|N|^{2}|| G \mid$. (Thus $K$ wr $K$ contains an isomorphic copy of every extension $G$ of $K$ by $K$.)

Proof: Let $\psi: G \rightarrow K$ be the natural homomorphism of $G$ onto $K$ with kernel $N$. Let $T:=\left\{t_{u} \mid u \in K\right\}$ be a set of right coset representatives of $N$ in $G$ such that

$$
\psi\left(t_{u}\right)=u
$$

for each $u \in K$. If $x \in G$, then

$$
\begin{aligned}
\psi\left(t_{u} x\right) & =\psi\left(t_{u}\right) \psi(x) \\
& =u \psi(x) \quad(\text { by }(18)) \\
& =\psi\left(t_{u \psi(x)}\right) \quad(\text { by }(18))
\end{aligned}
$$

Therefore, since $\psi\left(t_{u} x\right)=\psi\left(t_{u \psi(x)}\right)$ it follows that $\psi\left(t_{u} x\right) \psi^{-1}\left(t_{u \psi(x)}\right)=1$. Therefore, $\psi\left(t_{u} x t_{u \psi(x)}^{-1}\right)=1$. Thus $t_{u} x t_{u \psi(x)}^{-1}$ lies in the kernel $N$, that is, $t_{u} x t_{u \psi(x)}^{-1} \in N$. By the definition of wreath product, we can define a function for each $x \in G$, such that $f_{x}: K \rightarrow K$ by

$$
f_{x}(u):=t_{u} x t_{u \psi(x)}^{-1}
$$

for all $u \in K$.

Now $\phi\left(\left(f_{x}, \psi(x)\right)\right)=x \in G$ defines an embedding $\phi$ of $K$ wr $K$ into $G$ with the function (18).

We seek to show that $\phi$ is a homomorphism and injective, hence an embedding.

Take $x, y \in G$, then

$$
\phi\left(\left(f_{x}, \psi(x)\right)\right) \phi\left(\left(f_{y}, \psi(y)\right)\right)=x y=\phi\left(f_{x y}, \psi(x y)\right)
$$


Now since $\psi$ is a homomorphism,

$$
\psi(x) \psi(y)=\psi(x y)
$$

By (17), $f_{x y}$ can be expressed for all $u \in K$ as follows:

$$
\begin{aligned}
f_{x y}(u) t_{u \psi(x y)} & =t_{u} x y \quad \text { (by (19)) } \\
& =t_{u} x t_{u \psi(x)}^{-1} t_{u \psi(x)} y \quad(\text { by }(19)) \\
& =f_{x}(u) t_{u \psi(x)} y \\
& =f_{x}(u) t_{u \psi(x)} t_{u \psi(x) \psi(y)} t_{u \psi(x) \psi(y)} y \quad \text { (by (19)) } \\
& =f_{x}(u) f_{y}(u \psi(x)) t_{u \psi(x) \psi(y)} \quad \text { (by (6) and (20)) } \\
& =f_{x}(u) f_{y}^{\psi(x)^{-1}}(u) t_{u \psi(x y)} \quad \text { (by (6) and (20)) }
\end{aligned}
$$

Therefore, we have

$$
f_{x y}=f_{x} f_{y}^{\psi(x)^{-1}}
$$

Hence

$$
\begin{aligned}
& \phi\left(\left(f_{x}, \psi(x)\right)\right) \phi\left(\left(f_{y}, \psi(y)\right)\right) \\
& =\left(f_{x} f_{y}^{\psi(x)^{-1}}, \psi(x) \psi(y)\right)=\phi\left(\left(f_{x}, \psi(x)\right)\left(f_{y}, \psi(y)\right)\right)
\end{aligned}
$$

(21)), showing that $\phi$ is a homomorphism.

Next, we show that $\phi$ is injective. Now $\operatorname{ker} \phi=1$ since $\phi\left(\left(f_{x}, \psi(x)\right)\right)=1$ implies $x=1$ and so $f_{1}=1$ and $\psi(1)=1$, thus from Lagrange theorem, $\frac{|G|}{|K|^{K \mid}|K|}=\frac{|N|^{2}}{|G|}$, i.e. $|N|^{2}|| G \mid$ as $f_{1}=1$ and $\psi(1)=1$.

Finally, $\phi\left(\left(f_{x}, \psi(x)\right)\right)$ lies in $B$ when $\psi(x)=1$, and this happens exactly when $x \in N$.

Thus $K$ wr $K \lesssim G$.

\section{Example 4:}

$$
\begin{aligned}
& G=S_{4}=\{(1),(34),(23),(234),(243),(24),(12),(12)(34),(123), \\
& \text { Let } \quad(1234),(1243),(124),(132),(1342),(13),(134),(13)(24) \text {, and } \\
& \text { (1324),(1432),(142),(143),(14),(1423),(14)(23)\} } \\
& N=A_{4}=\{(1),(234),(243),(12)(34),(123),(124),(132) \text {, } \\
& \text { (134),(13)(24),(142),(143),(14)(23)\} } \\
& K=G / N=S_{4} / A_{4} \\
& =\{\{(1),(234),(243),(12)(34),(123),(124),(132),(134),(13)(24),(142) \text {, } \\
& (143),(14)(23)\},\{(34),(23),(24),(12),(1234),(1243),(1342),(13), \quad \text {. Now } \\
& \text { (1324),(1432),(14),(1423)\}\} } \\
& \cong C_{2} \\
& \frac{|N|^{2}}{|G|}=\frac{12^{2}}{24}=6 \text {. Then the wreath product }
\end{aligned}
$$


$K w r K \cong C_{2} w r C_{2}$ $=\{(1),(34),(12),(12)(34),(13)(24),(1324),(1423),(14)(23)\}$ which is a group of order 8.

Remark 5: If $\phi: G \rightarrow H$ is an embedding, then $n|G|=|H|$ where $n \neq 1 \in \mathbb{N}$ is the index of $G$ by $H$. If the index $n=1$, then $\phi$ is as isomorphism.

Theorem 6 (Dixon \& Mortimer, 1996): (Universal Embedding Theorem)

Let $G$ be an arbitrary group with a normal subgroup $N$, and put $K:=G / N$. Then there is an embedding $\phi: G \rightarrow N$ wr $K$ such that $\phi$ maps $N$ onto $\operatorname{Im} \phi \cap B$ where $B$ is the base group of $N w r K$. (Thus $N w r K$ contains an isomorphic copy of every extension $G$ of $N$ by $K$.)

Condition 1: If $G$ is of order $p$, i.e. $|G|=p$, then $|N|=p$ or 1 , by Lagrange's theorem, then either $G=N$ or $N=\{e\}$.

If $|N|=p$, then $|G|=|N|=p$, then $G=N$ and so $K=G / N=G / G \cong\{e\}$. Thus $|K|=1$, and $\phi$ being a homomorphism implies that $n|G|=|N|^{|K|}|K|$ and so $n=1$.

If $|N|=1$, then $N=\{e\}$ and so $K=G / N=G /\{e\} \cong G$. Thus $|K|=|G|=p$, and $\phi$ being a homomorphism implies that $n|G|=|N|^{|K|}|K|$ and so $n=1$.

Thus $\phi$ is an isomorphism if $|G|=p$ or rather $\phi$ is an isomorphism if $G$ is cyclic.

Condition 2: If the order of $G$ is $p^{2}$, then $G$ is known to be an Abelian group and by Lagrange's theorem $|N|=p^{2}, p$ or 1 .

If $|N|=p^{2}$, then $G=N$ and so $|K|=|G / N|=|G| /|N|=p^{2} / p^{2}=1$. Thus $K=\{e\}$. And $\phi$ being a homomorphism implies that $n|G|=|N|^{|K|}|K|$ and so $n p^{2}=p^{2}$ and $n=1$. Therefore, $\phi$ is an isomorphism if $G=N$.

If $|N|=p$, then $|K|=|G / N|=|G| /|N|=p^{2} / p=p$. Thus $\phi$ being a homomorphism implies that $n|G|=|N|^{|K|}|K|$ and so $n p^{2}=p^{p} p$ and $n=p^{p-1}$ the index.

If $|N|=1$, then $N=\{e\}$ and so $|K|=|G / N|=|G| /|N|=p^{2} / 1=p^{2}$. Thus $\phi$ being a homomorphism implies that $n|G|=|N|^{|K|}|K|$ and so $n p^{2}=1^{p^{2}} p^{2}=p^{2}$. Thus $n=1$. Therefore, $\phi$ is an isomorphism if $N=\{e\}$.

Condition 3: If $G$ is a simple group, then either $N=G$ or $N=\{e\}$.

If $N=G$, then $|N|=|G|$, then $|K|=|G| /|N|=1$, and $\phi$ being a homomorphism implies that $n|G|=|N|^{|K|}|K|$ and so $n=1$.

If $N=\{e\}$, then $|N|=1$, then $|K|=|G| / 1=|G|$, and $\phi$ being a homomorphism implies that $n|G|=|N|^{|K|}|K|$ and so $n=1$.

Thus $\phi$ is an isomorphism if $G$ is simple.

\section{Example 5:}

Let $G=\langle(12345)\rangle=\{(1),(12345),(13524),(14253),(15432)\}$. Then the normal subgroup $N=G$ or $N=\{(1)\}$. If $N=G$, then $K=G / N=G / G=\{\{(1),(12345),(13524),(14253),(15432)\}\} \cong\{(1)\}$. Thus $N$ wr $K \cong\{(1),(12345),(13524),(14253),(15432)\}=G$. If $N=\{(1)\}$, then $K=G / N=G /\{(1)\}=\{(1),(12345),(13524),(14253),(15432)\}=G$. Thus $N$ wr $K \cong\{(1),(12345),(13524),(14253),(15432)\}=G$. Thus an isomorphism 
as $G$ is Cyclic, simple and its order is prime.

\section{Discussion}

Embedding is an important property of wreath product as it helps in preserving structures between groups. Under some conditions we have seen that the Universal embedding Theorem is an isomorphism.

\section{Conclusion}

In this paper, we were able to give a new proof of the theorem by Audu (1991), which proved that a group can be embedded into the wreath product of a factor group by a normal subgroup and also proved that a factor group can be embedded inside a wreath product and the wreath product of a factor group by a factor group can be embedded into a group. It was shown that when the abstract group in the universal embedding theorem is a p-group, cyclic and simple, the embedding is an isomorphism.

\section{Conflicts of Interest}

The authors declare no conflicts of interest regarding the publication of this paper.

\section{References}

[1] Audu, M.S. (1991) On Transitive Permutation Groups. Afrika Mathematika, Journal of African Mathematical Union, 4, 155-160.

[2] Suzuki, M. (1982) Group Theory, I. Grundlagen der mathematischen Wissenshaften 247. Springer, Berlin. https://doi.org/10.1007/978-3-642-61804-8

[3] Dixon, J.D. and Mortimer, B. (1996) Permutation Groups. Springer-Verlag, Berlin, New York. https://doi.org/10.1007/978-1-4612-0731-3

[4] Conway, J.H., Hulpke, A. and McKay, J. (1998) On Transitive Permutation Groups. LMS Journal of Computation and Mathematics, 1, 1-8.

https://doi.org/10.1112/S1461157000000115

[5] Bamberg, J. (2005) Permutation Group Theory. https://faculty.psau.edu.sa/filedownload/doc-6-pdf-3b66c4570863b65a0f844ce98259 68f9-original.pdf

[6] Chan, M. (2006) The Distinguishing Number of the Direct Product and Wreath Product Action. Journal of Algebraic Combinatorics, 24, 331-345.

https://doi.org/10.1007/s10801-006-0006-7

[7] Cameron, P.J. (2019) Primitivity, Combinatorics, Algebra and More. http://www-groups.mcs.st-andrews.ac.uk/ pjc/talks/camconf/pjc_cam2.pdf

[8] Tamuli, B.K. (1972) Groups and the wreath Product. Ph.D. Thesis, Gauhati University, India.

[9] Mikaelian, V.H. (2002) Two Problems on Varieties of Groups Generated by Wreath Products. IJMMS, 31, 65-75. https://doi.org/10.1155/S0161171202012528

[10] Hulpke, A. (2004) Constructing Transitive Permutation Groups. Journal of Symbolic Computation, 39, 1-3. https://doi.org/10.1016/j.jsc.2004.08.002

[11] Fraleigh, J.B. (2005) A First Course in Abstract Algebra. 6th Edition, Addison Wes- 
ley Longman, NJ.

[12] Ibrahim, A.A. and Audu, M.S. (2007) On Wreath Product of Permutation Groups. Universidad Católica del Norte, Antofagasta-Chile, 26, 73-90. 